\title{
Differential glatiramer acetate treatment persistence in treatment-naive patients compared to patients previously treated with interferon
}

Mireya Fernández-Fournier ${ }^{1 *}$, Antonio Tallón-Barranco ${ }^{1}$, Beatriz Chamorro ${ }^{1}$, Patricia Martínez-Sánchez² and Inmaculada Puertas ${ }^{1}$

\begin{abstract}
Background: In the treatment of multiple sclerosis, a change of therapy is considered after treatment failure or adverse events. Although disease modifying drugs' (DMD) efficacy and side effects have been fully analysed in clinical trials, the effects of previous therapy use are less well studied. We aimed to study medication persistence with glatiramer acetate in treatment-naive patients and in patients previously treated with interferon.

Methods: A retrospective study of relapsing-remitting multiple sclerosis patients treated with glatiramer acetate in an MS Unit of a Spanish University Hospital (January 2004 - September 2013). Treatment time on glatiramer acetate was studied. Reasons for treatment discontinuation were considered as follows: lack of efficacy, serious adverse event, injection-related side effect, pregnancy and lost to follow-up. Use of prior DMD was registered and analysed. Homogeneity of groups was analysed using Fisher's and Mann-Whitney's tests. The Kaplan Meier method and Cox regression model were used to estimate time to and risk of treatment discontinuation.

Results: In total, 155 relapsing-remitting multiple sclerosis patients were treated with glatiramer acetate: 100 treatmentnaive patients and 55 treated previously with interferon. At the end of the study, 76 patients (49.0\%) continued on glatiramer acetate (with an average treatment time (ATT) of 50.4 months, s.d.32.8) and 50 patients (32.3 \%) had switched therapy: 27 patients (17.4\%) for inefficacy (ATT 29.2 months, s.d.17.5), 20 patients (12.9\%) for injection site reactions (ATT 16.5 months, s.d.20.3) and 3 patients (1.9\%) after serious adverse events (ATT 15.7 months, s.d.15.1). ATT in our cohort was 39 months (s.d.30.0), median follow-up 34 months. Six months after glatiramer acetate initiation, probability of persisting on GA was $91.4 \%, 82.5 \%$ after 12 months and $72.5 \%$ after 2 years. The risk of glatiramer acetate treatment discontinuation was 2.8 [1.7 - 4.8] times greater for treatment-naive patients than for patients treated previously with interferon and this was hardly modified after adjusting for sex and age.

Conclusions: Glatiramer acetate was safe and useful with low rates of serious adverse events and low rates of break-through disease. Injection intolerance proved a major limitation to glatiramer acetate use. Patients who had been previously treated with interferons presented a lower probability of glatiramer acetate discontinuation than treatment-naive patients.
\end{abstract}

\footnotetext{
* Correspondence: fernandezfournier@hotmail.com

${ }^{1}$ Clinical Neuroimmunology and Multiple Sclerosis Unit, La Paz University

Hospital, Madrid, Spain

Full list of author information is available at the end of the article
}

\section{Biomed Central}

(C) 2015 Fernández-Fournier et al. Open Access This article is distributed under the terms of the Creative Commons Attribution 4.0 International License (http://creativecommons.org/licenses/by/4.0/), which permits unrestricted use, distribution, and reproduction in any medium, provided you give appropriate credit to the original author(s) and the source, provide a link to the Creative Commons license, and indicate if changes were made. The Creative Commons Public Domain Dedication waiver (http://creativecommons.org/publicdomain/zero/1.0/) applies to the data made available in this article, unless otherwise stated. 


\section{Background}

When treating multiple sclerosis (MS) patients, the main goal is to slow disease progression and improve healthrelated quality of life [1]. Selecting an optimal individualized treatment for MS patients is becoming progressively more complicated as treatment options and their side effects increase [2, 3].

Both interferons (IFNs) and glatiramer acetate (GA) are injectable disease modifying drugs (DMDs) approved and used as first-line therapies in MS. If disease activity is still observed despite treatment or if adverse events occur, a therapy switch is considered. Some studies conclude that switching between injectable DMDs, mainly from IFNs to GA, can result in a substantial relapse rate reduction, both for patients switched for treatment failure as well as for those who are switched for other reasons [4-8].

Although DMD efficacy and side effects have been fully studied in clinical trials, the effects of previous therapy use are less well known. Analysing experience gathered from 'real-world' clinical practice could help make rational individualised treatment decisions. Treatment persistence with GA when used after treatment failure with IFNs is not well characterized. The aim of this pilot exploratory study was to describe GA use and treatment persistence, measured as time to GA treatment discontinuation, in a group of Relapsing Remitting MS (RRMS) treatment-naive patients and in a group of RRMS patients who had previously received treatment with IFNs.

\section{Methods}

\section{Study design}

An independent, retrospective observational study was conducted at an MS Unit of a tertiary University Hospital. Clinical records were used to identify patients who had been prescribed glatiramer acetate (GA) between January 1st 2004 and October 1st 2013. Clinical diagnosis, prior DMD treatment, dates of GA treatment initiation and end, as well as reasons for GA treatment discontinuation, were studied. Patients were included according to their clinical history in one of the following groups: treatment-naive patients and patients treated previously with IFN. All RRMS patients who had been exposed to IFN before initiating treatment with GA were included.

Treatment failure was defined as reasons given by the treating neurologist for GA discontinuation. The following reasons were considered: lack of efficacy (including relapses, progression of disability or magnetic resonance imaging (MRI) activity), occurrence of serious adverse event (SAE), injection site reactions, pregnancy or pregnancy planning at study end-point and loss to follow-up. Time to treatment discontinuation was studied. End of observation period was October 1st 2013. Treatment time for each individual patient was calculated in months as the time lapse between GA treatment initiation and the last documented injection. Average treatment times (ATT) were calculated for each group.

\section{Patients}

The study was conducted at the Neurology Department of La Paz University Hospital in Madrid. In total 155 patients met inclusion criteria: age $\geq 18$ years, RRMS diagnosis according to 2010 revised McDonald criteria [9] and treatment with GA between January $1^{\text {st }} 2004$ - October $1^{\text {st }}$ 2013. Patients were excluded if another diagnosis different from MS was given on follow-up. Patients were then excluded from statistical analysis if data regarding GA initiation and end dates was missing.

Throughout the study period DMD initiation and change were performed at the discretion of the MS Unit medical team following usual clinical practice $[3,10,11]$. A lack of efficacy or suboptimal response to GA was considered the reason for a treatment change based on the patient's clinical history, considering the occurrence of relapses ( $\geq 2$ relapses or 1 severe relapse), accrual of disability or disease activity on follow-up MRI (presence of gadolinium-enhancing lesions or increased T2-lesion load, including both new and enlarging T2-lesions). Clinical, including EDSS scores, DMD initiation and end dates, and MRI data were collected and stored in an electronic database. All patients underwent regular follow-up consisting of six-monthly neurological assessments and laboratory testing including liver and thyroid gland function. After therapy approval by the health authorities, all patients received training sessions on self-injectable DMD administration by a nurse who specialised in the care of MS patients. In the training sessions realistic expectations were established, side effects were anticipated, as well as their management, and administration technique was taught. After training, patients self-injected the DMD at home. If they had questions, patients could use a telephone help-line staffed by nurses and neurologists. Unscheduled visits were performed for the assessment and treatment of side effects, relapses or any other clinically relevant condition. This study is a retrospective analysis of our MS clinical data, and has been conducted using data that was anonymized for analysis so specific written informed consent was not obtained from patients. This study has been performed according to the regulations of the Ethics Committee of La Paz University Hospital.

\section{Data analysis}

Statistical analysis was performed using SAS 93 (SAS Institute, Cary, NC, USA). Quantitative data are described using the mean \pm standard deviation ( \pm s.d.), median and range. Qualitative data are described using absolute frequencies and percentages. The homogeneity of the groups was analysed using Fisher's exact test for 
categorical data and Mann-Whitney's test for quantitative data. Treatment time on GA was studied. The Kaplan Meier method was used to estimate time to treatment discontinuation. Risk of treatment discontinuation was estimated using a Cox regression model, with and without adjusting for sex and age at the time of GA initiation. The independent variable of this study was time to GA treatment discontinuation, defined as the time lapse between treatment initiation and treatment discontinuation in months. Patients who were still treated with GA on October 1st 2013 and those lost to follow up on treatment with GA have been considered censored values as per survival analysis. Estimated Hazard ratios with their 95 \% confidence intervals are provided.

\section{Results}

Between January 1st 2004 and October 1st 2013, 155 RRMS patients ( $68.3 \%$ females) who were treatmentnaive (100 patients) or had been previously treated with IFNs (55 patients) were prescribed GA at the Neuroimmunology and MS Unit of La Paz University Hospital. Ages at GA treatment initiation ranged from 17 to 60 years of age, average $37( \pm 9)$ and were similar for both treatment groups (treatment-naive patients and patients treated previously with IFN). There was a tendency for a greater proportion of females in the treatment-naive group (73 \% vs. $58.1 \%, P=0.073$ ). Median EDSS at GA prescription was similar for both groups (Table 1).

At the end of the study (Fig. 1), from the 155 patients, 76 patients $(49.0 \%)$ were still being treated with GA. Prescription had been withdrawn in 50 cases (32.3\%), 3 patients $(1.9 \%)$ had temporarily stopped GA for pregnancy planning and 26 patients (16.8 \%) had been lost to followup (Fig. 2). From the 50 patients who stopped GA, all patients with previous IFN exposure escalated therapy to second line drugs, while those in the treatment-naive group followed a variety of pathways, including switching to second-line drugs (60.4\% patients), but also to IFN (29.2 \%) and immunosuppressive drugs (4.2\%), as well as stopping treatment altogether following patients' demands ( $4.2 \%)$ or conversion to secondary progressive MS (2.1\%).

In our cohort, reasons for stopping GA treatment were: (a) lack of efficacy, including relapses (22 patients, $14.2 \%)$, disability accrual (3 patients, $1.9 \%$ ) or evidence of disease activity on follow-up MRI (2 patients, $1.3 \%$ ); (b) injection intolerance (20 patients, $12.9 \%$ ): mainly injection pain and mild-moderate skin reactions such as redness or itching; (c) SAEs (3 patients, $1.9 \%$ ): injection associated unremitting chest pain, allergic reactions or severe local reactions such as marked lipoatrophy. Amongst patients who discontinued GA due to injection intolerance, most ( $n=19$ patients, $95 \%$ ) belonged to the treatment-naive group.

Overall, patients in our cohort were treated with GA an average of 39 months (s.d 30.0). Median follow-up was 34 months. Six months after GA treatment initiation, probability of continuing on GA was $91.4 \%$ (88.6\% for treatment-naive patients and $96.3 \%$ for patients previously treated with IFN). Twelve months after treatment initiation, probability of continuing on GA was $82.5 \%$ (76.8 \% for treatment-naive patients and $92.5 \%$ for patients previously treated with IFN). Two years after treatment initiation, probability of continuing on GA was $72.5 \%$ (62.3\% for treatmentnaive patients and $90.6 \%$ for patients previously treated with IFN). Treatment-naive patients were found to be significantly more prone to discontinue GA than those with previous exposure to IFNs (Fig. 3). The risk of GA discontinuation was 2.8 [C.I. $95 \%$ : 1.7 - 4.8] times greater for treatment-naive patients and this was hardly modified after adjusting for sex and age (3.0 [C.I. $95 \%: 1.8-5.2]$ ).

Table 1 Characteristics of patients treated with glatiramer acetate (GA), January 2004 - October 2013

\begin{tabular}{|c|c|c|c|c|}
\hline & Total patients $(n=155)$ & Treatment-naive $(n=100)$ & Previous treatment with interferon $(n=55)$ & $P$ \\
\hline Age: range, y & $17-60$ & $17-56$ & $24-60$ & $p=0.3$ \\
\hline Mean $( \pm$ s.d.) & $37( \pm 9)$ & $37( \pm 9)$ & $38( \pm 8)$ & \\
\hline Female sex, $n(\%)$ & $106(68.3)$ & $73(73.0)$ & $32(58.1)$ & $p=0.07$ \\
\hline \multicolumn{5}{|l|}{ Geographical origin } \\
\hline South Europe (Spain), n (\%) & $145(93.5)$ & $94(94.0)$ & $51(92.7)$ & \\
\hline Eastern Europe, $n(\%)$ & & $2(2.0)$ & $1(1.8)$ & \\
\hline North Africa, $n(\%)$ & & $2(2.0)$ & $1(1.8)$ & \\
\hline South America, $n$ (\%) & & $2(2.0)$ & $2(3,6)$ & \\
\hline Time to treatment ${ }^{\mathrm{a}}$ : Mean $( \pm$ s.d.), y & $5.2( \pm 5.9)$ & $2.7( \pm 5.5)$ & $6.7( \pm 5.7)$ & $p<0.001$ \\
\hline EDSS at GA initiation (median [IQR]) & & $1.5[1-3]^{b}$ & $2[1-3]^{b}$ & $p=0.2$ \\
\hline
\end{tabular}

y years, s.d standard deviation, GA Glatiramer Acetate, IQR Interquartile Range

${ }^{a}$ Time to treatment $=$ Average time-lapse from diagnosis to GA treatment initiation

${ }^{b}$ EDSS at GA initiation data availability of $55 \%$ 


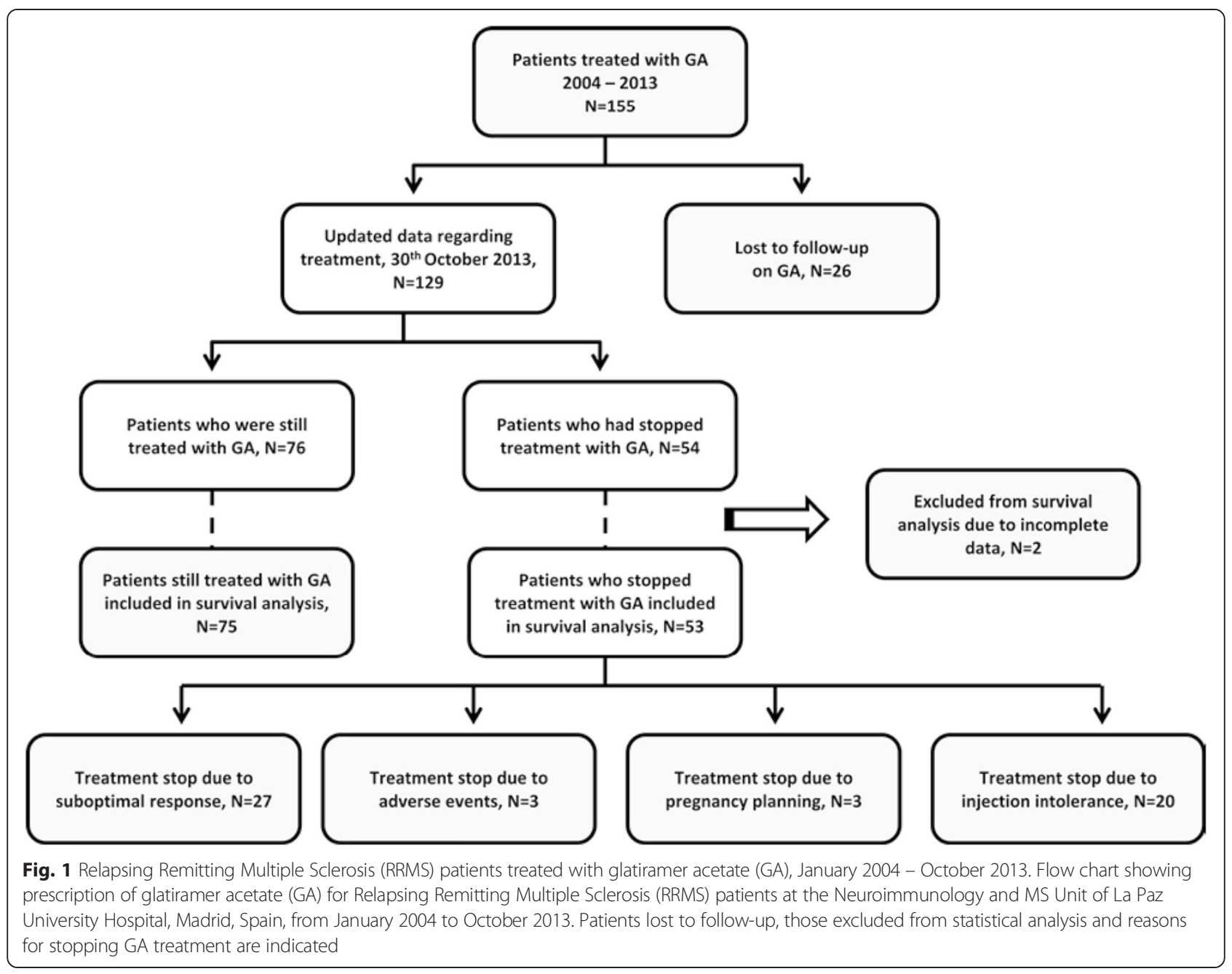

Regarding time spent on GA, ATT for patients who persisted on GA at the end of the study was 50.4 months (s.d. 32.8). Patients who discontinued GA for lack of efficacy had an ATT of 29.2 months (s.d. 17.5), amongst patients discontinuing for SAEs, ATT was15.7 months (s.d. 15.1) and for injection site reactions ATT was 16.5 months (s.d. 20.3). Amongst patients lost to followup, ATT was 37.8 months (s.d. 26.3). ATT for patients discontinuing GA for pregnancy planning was 29.7 months (s.d. 6.0) (Fig. 4).

\section{Discussion}

We present an independent, observational study analysing treatment persistence on glatiramer acetate (GA) in an MS Unit of a Spanish tertiary University Hospital. Little is known on how the use of previous DMDs affects treatment outcome. This is the second published study that aims to describe differences in treatment persistence with GA in treatment-naive patients with respect to patients previously treated with IFNs, and the first to do so in Europe.
Subcutaneous GA is a well-established treatment for RRMS. Although GA's exact mechanism of action remains to be fully elucidated, key roles seem to be modulation of inflammatory response and neuroprotective effects [12]. Clinical trials and follow-up studies have shown that GA reduces clinical and radiological disease activity, proves more effective than placebo and shows at least similar efficacy to subcutaneous IFN $\beta-1 \mathrm{a}$ and IFN $\beta-1 \mathrm{~b}$ [12-18]. In the literature, GA is described to be effective both in treatmentnaive patients and in those switching from a previous DMD because of lack of efficacy or side effects [19].

The current paradigm is to start RRMS treatment with injectable DMDs as a first-line therapy and then advance into the therapeutic pyramid until the disease is effectively controlled. However, first-line treatment failure with IFNs may be due to the existence of neutralizing antibodies [12], poor tolerance or occurrence of adverse events, and may not reflect the need to escalate to second-line therapies such as Fingolimod or Natalizumab. Second-line drugs offer potentially greater efficacy, but are associated with an increased level of risk [3, 


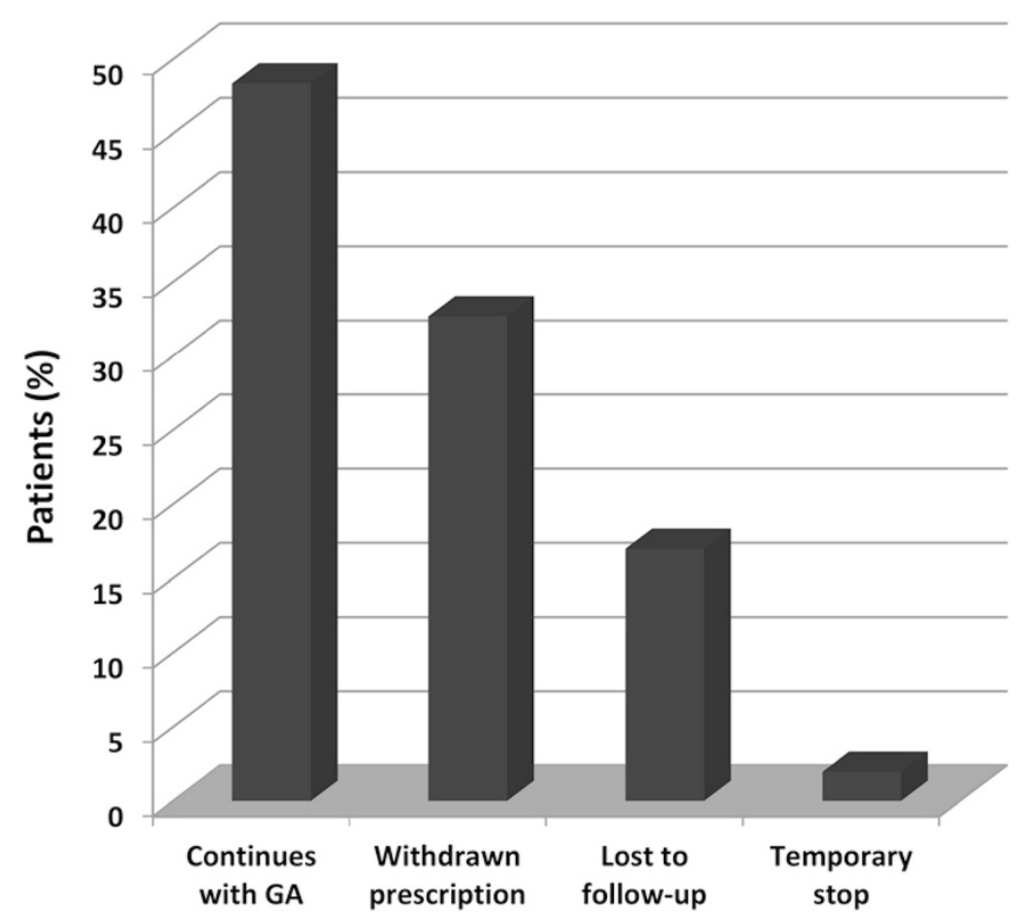

Fig. 2 Proportion of patients in each treatment persistence group. Graph showing proportion of patients according to different glatiramer acetate (GA) treatment persistence groups at the Neuroimmunology and MS Unit of La Paz University Hospital on October 1st 2013

20], also costs sometimes need to be taken into account when evaluating treatment options. Thus, changes between first-line agents may be, in some cases, a suitable option.

In contrast to the situation in clinical trials, switching between DMDs is common in daily practice. Studying treatment use and response in 'real-world' clinical practice is relevant for the future care of RRMS. It is important to address questions not answered by clinical trials regarding not only efficacy, but also tolerability and treatment persistence [21]. Treatment persistence varies

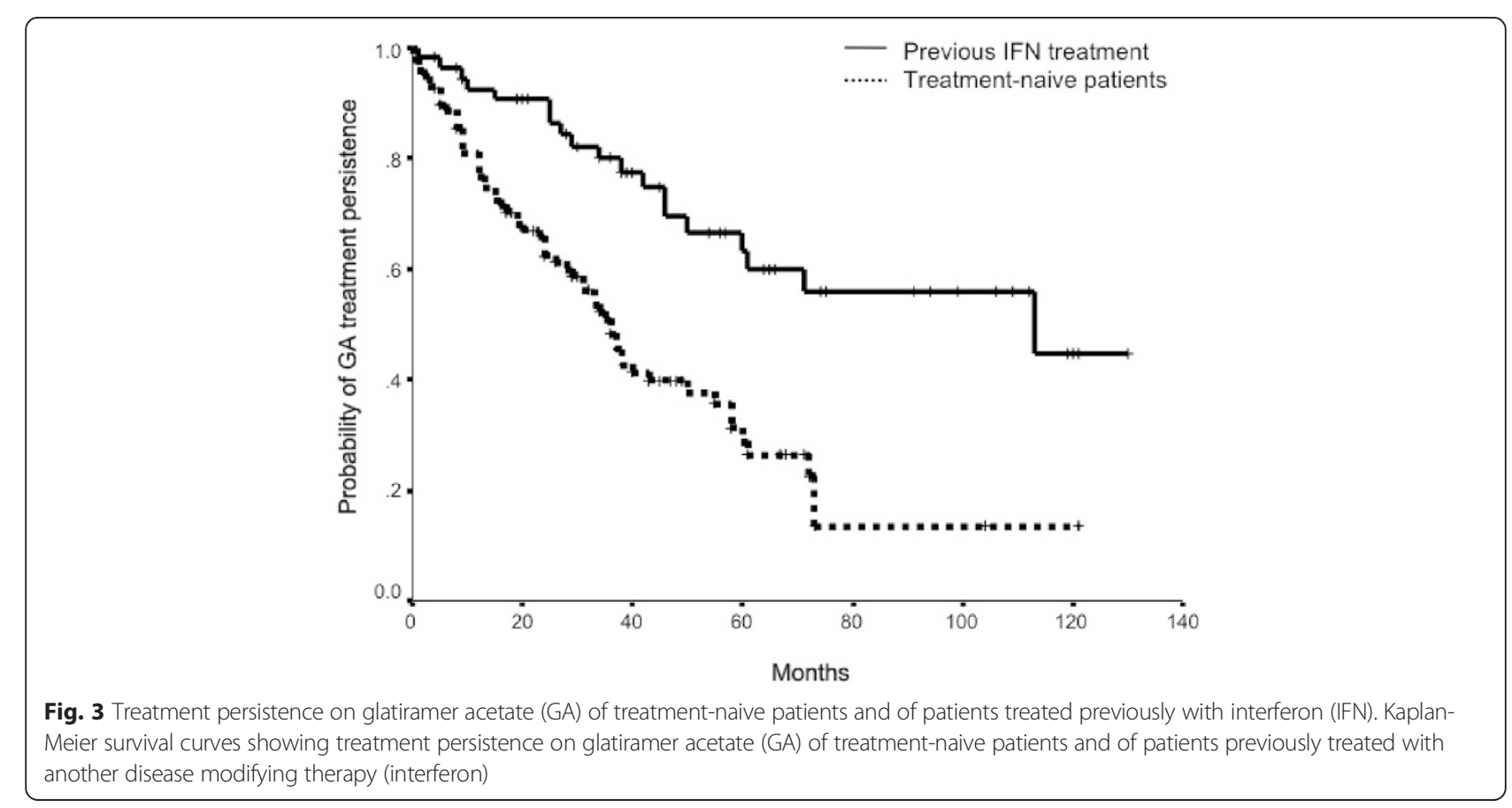




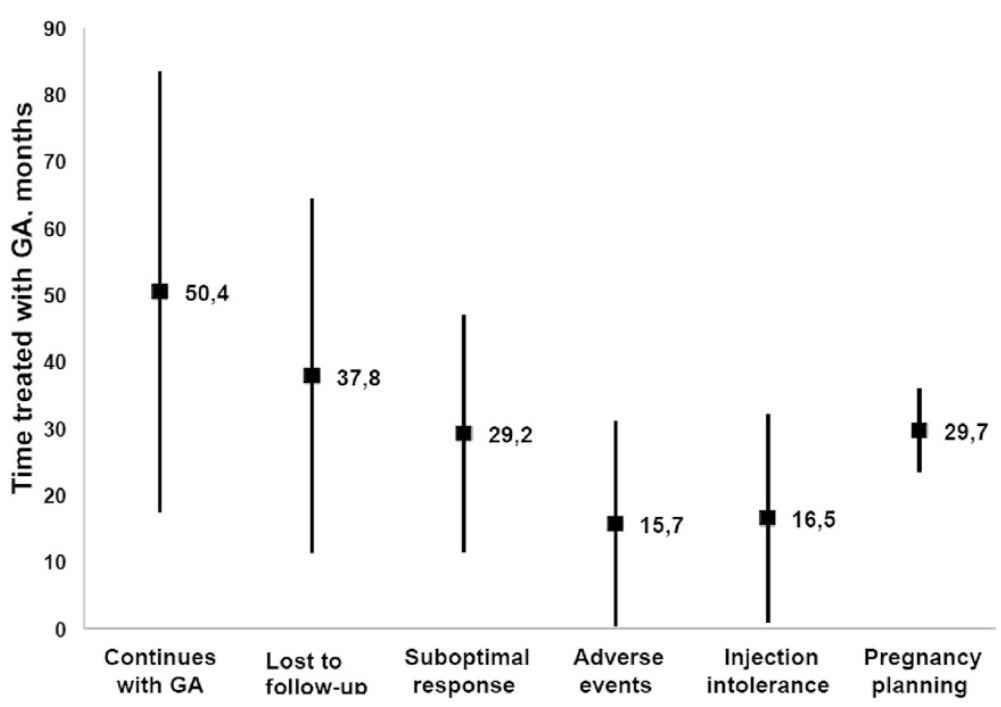

Fig. 4 Average treatment-time with glatiramer acetate (GA) according to patient's treatment persistence group. Graph showing average treatmenttime (ATT) in months ( \pm standard deviation) with glatiramer acetate (GA) at the Neuroimmunology and MS Unit of La Paz University Hospital between January 2004 and October 2013, according to patient's GA treatment persistence category

with country of residence [22]. Jokubaitis et al. compared treatment persistence on GA and IFN in Australian patients and report that, in their cohort, patients receiving IFN $\beta-1 \mathrm{a}$ as a first DMD persisted longer on treatment than those treated with GA as a first DMD [22]. Similarly, Kleinman et al. found lower discontinuation rates on IFN $\beta$ than on GA in the US [23]. On the other hand, data from the REGARD clinical trial do not show significant differences in treatment persistence between IFN $\beta-1 a$ and GA [24]. In the Australian cohort, higher EDSS and younger age at DMD initiation were predictive of treatment discontinuation, however in our cohort, adjusting for age hardly modified the relative risk of GA discontinuation. Another study on DMD use reported female sex as a predictive factor of treatment discontinuation [25], but this was not reproduced in the Australian study or in our cohort. Missing data do not allow us to conclude if EDSS is predictive of treatment discontinuation in our cohort.

With respect to efficacy, GA was useful in our 'real world' setting, with only $17.4 \%$ of patients presenting breakthrough disease (relapses, progression of disability or MRI activity), which is lower than that of clinical trials and on the low end of reported breakthrough disease for clinical practice, which is of $18-30 \%$ in 5 years for first-line DMDs [19]. This difference might be explained by clinical trials' selective inclusion criteria that tend to include only highly active patients with a greater probability of break-through disease than that of "the average MS patient". It may also result from underestimating real breakthrough activity, as patients who might have remained on GA despite disease activity are not included.
Regarding safety and tolerability, subcutaneous GA was generally well tolerated in clinical trials and extension studies, the most commonly reported adverse event being injection site reactions and mild vasodilation [12] (http://www.copaxone.com/about-copaxone/copaxonesafety-and-effectiveness, cited 2014 Mar 14). Our results agree with clinical trials regarding treatment safety, only $1.9 \%$ patients developed relevant medical side-effects (injection associated unremitting chest pain, allergic reactions or severe local reactions such as marked lipoatrophy). However, our data show a relatively high treatment discontinuation rate (12.9\%) due to injection intolerance (mainly injection pain and mild-moderate skin reactions, including redness and itching), mostly amongst treatment-naive patients, and this was independent of gender. Along these lines, previous studies indicate that the lack of treatment tolerance is a major reason for DMD discontinuation [22, 26, 27].

The overall risk of GA treatment discontinuation in our cohort was almost 3 times greater for treatmentnaive patients than for patients treated previously with IFN. Similarly, Jokubaitis et al. report lower treatment persistence on GA than on IFN only amongst treatmentnaive patients and not when these drugs were used as second therapies [22]. We believe this might be influenced by non-naive patients being used to self-injections.

Patients who discontinue treatment with IFNs may do so because of side-effects or disease activity. With regard to risk-benefit ratios, MS disease activity of some patients may not justify assuming the potential risks of second-line drugs. This study argues in favour of 
incorporating GA in the MS therapeutic algorithm not only as an initial agent but as a second therapy for certain patients who discontinue treatment with IFNs. Further investigation regarding reasons for treatment discontinuation and comparisons between treatment-naive patients and switchers is required.

Limitations to our study include the retrospective design that limits study variables. The prevalence of IFN neutralizing antibodies was not consistently checked, treatment compliance was not well recorded and no questionnaire addressing patients' perception of treatment was administered. Other limitations of this study are cohort size and loss to follow-up, with 26 patients $(16.8 \%)$ lost to follow-up on treatment with GA, however this seems reasonable taking into account the 10 year time-span of the study. This pilot study was designed as an exploratory study so we were not able to conduct certain sub-analysis. We were not able to eliminate potential bias by adjusting for disability which can influence treatment persistence [22, 25]. However, despite the limited sample size, significant results are obtained in treatment persistence between treatment-naive patients and patients treated previously with IFN. Regarding the clinical applicability of these results, the time frame must be taken into account as it may influence treatment persistence. In our cohort, patients who discontinued GA for lack of treatment efficacy were on GA for over two years (29.2 months $( \pm 17.5))$. Patients underwent regular followup visits at least twice per year, so GA might be seen a useful initial treatment even if long-term response is suboptimal. However, patients might have remained on GA waiting for the arrival of new therapies. Between 2004 and 2013 progress in the treatment of MS was remarkable, with new second-line drugs being licensed. At the same time, MRI criteria for early identification of non-responders were developed and progressively incorporated into clinical practice. With regard to the future, the situation is one of an increasing complexity, as new oral drugs, available as firstline therapies, arrive [20,28].

A recent review comparing comparing the efficacy of IFN with that of GA in RRMS concludes that, even if safety and efficacy parameters of both drugs are similar, patient needs such as different levels of tolerability need to be addressed and taken into account for treatment choice [29]. A prospective study with questionnaires specifically addressing subjective perception of treatments as well as treatment adherence, in both treatment-naive patients and switchers, might shed light on the differential treatment persistence.

\section{Conclusions}

In summary, GA was safe and useful with low rates of serious adverse events, similarly to previously published data, and low rates of break-through disease. Injection intolerance proved a major limitation to GA use, mostly amongst treatment-naive patients. We conclude that prior DMD use seems to influence persistence on GA; patients with previous exposure to IFN presented a significantly lower probability of GA treatment discontinuation than treatment-naive patients.

\section{Abbreviations}

ATT: Average treatment time; DMD: Disease modifying drug; GA: Glatiramer acetate; IFN: Interferon; MRI: Magnetic resonance imaging; MS: Multiple sclerosis; RRMS: Relapsing remitting multiple sclerosis; SAE: Serious adverse event; s.d: Standard deviation.

\section{Competing interests}

The authors declare that they have no competing interests.

\section{Authors' contributions}

MFF contributed to data analysis and interpretation, as well as drafting the manuscript. ATB participated in study conception and design as well as revising the manuscript for important intellectual content. BC contributed to acquisition of data as well as its analysis and interpretation. PMS participated in data analysis and critical revision of the manuscript. IP has been involved in critically revising the manuscript for important intellectual content. All authors agree to be accountable for all aspects of the work in ensuring that questions related to the accuracy or integrity of any part of the work are appropriately investigated and resolved.

\section{Acknowledgements}

We thank the Neurology Department at La Paz University Hospital. We also thank Rosario Madero from the Department of Statistics for technical assistance. We also want to thank Teva Pharma S.L.U who, when asked, agreed to pay for the article processing charges.

\section{Author details}

${ }^{1}$ Clinical Neuroimmunology and Multiple Sclerosis Unit, La Paz University Hospital, Madrid, Spain. ²Department of Neurology, La Paz University Hospital, Madrid, Spain.

Received: 16 December 2014 Accepted: 5 August 2015

Published online: 19 August 2015

\section{References}

1. Miller JR. The importance of early diagnosis of multiple sclerosis. J Manag Care Pharm. 2004;10(3 Suppl B):S4-11.

2. Perrin Ross A. Management of multiple sclerosis. Am J Manag Care. 2013;19(16 Suppl):s301-6

3. Río J, Comabella M, Montalban X. Multiple sclerosis: current treatment algorithms. Curr Opin Neurol. 2011;24:230-7.

4. Caon C, Din M, Ching W, Tselis A, Lisak R, Khan O. Clinical course after change of immunomodulating therapy in relapsing-remitting multiple sclerosis. Eur J Neurol. 2006;13:471-4.

5. Carrá A, Onaha P, Luetic G, Burgos M, Crespo E, Deri N, et al. Therapeutic outcome 3 years after switching of immunomodulatory therapies in patients with relapsing-remitting multiple sclerosis in Argentina. Eur J Neurol. 2008;15:386-93.

6. Gajofatto A, Bacchetti P, Grimes B, High A, Waubant E. Switching first-line disease-modifying therapy after failure: impact on the course of relapsingremitting multiple sclerosis. Mult Scler. 2009;15:50-8.

7. Zwibel HL. Glatiramer acetate in treatment-naïve and prior interferon-beta1b-treated multiple sclerosis patients. Acta Neurol Scand. 2006;113:378-86

8. Río J, Tintoré M, Sastre-Garriga J, Nos C, Castilló J, Tur C, et al. Change in the clinical activity of multiple sclerosis after treatment switch for suboptimal response. Eur J Neurol. 2012;19:899-904.

9. Polman CH, Reingold SC, Banwell B, Clanet M, Cohen JA, Filippi M, et al. Diagnostic criteria for multiple sclerosis: 2010 revisions to the McDonald criteria. Ann Neurol. 2011;69:292-302.

10. Freedman MS. "Time is brain" also in multiple sclerosis. Mult Scler. 2009;15:1133-4

11. Group IW, Optimization T. Treatment optimization in multiple sclerosis: report of an international consensus meeting. Eur J Neurol. 2004;11:43-7. 
12. Scott LJ. Glatiramer acetate: a review of its use in patients with relapsingremitting multiple sclerosis and in delaying the onset of clinically definite multiple sclerosis. CNS Drugs. 2013;27:971-88.

13. Johnson KP. Glatiramer acetate for treatment of relapsing-remitting multiple sclerosis. Expert Rev Neurother. 2012;12:371-84.

14. Lublin FD, Cofield SS, Cutter GR, Conwit R, Narayana PA, Nelson F, et al. Randomized study combining interferon and glatiramer acetate in multiple sclerosis. Ann Neurol. 2013;73:327-40.

15. Fernández-Fernández Ó, García-Trujillo L, Guerrero-Fernández M, León A, López-Madrona JC, Alonso A, et al. Efectividad del acetato de glatiramero en la práctica clínica: un estudio observacional. Rev Neurol. 2012;54:1-9.

16. Carter NJ, Keating GM. Glatiramer acetate: a review of its use in relapsingremitting multiple sclerosis and in delaying the onset of clinically definite multiple sclerosis. Drugs. 2010 Aug 20;70(12):1545-77.

17. La Mantia L, Di Pietrantonj C, Rovaris M, Rigon G, Frau S, Berardo F, et al. Interferons-beta versus glatiramer acetate for relapsing-remitting multiple sclerosis. Cochrane database Syst Rev. 2014;7:CD009333.

18. Kalincik T, Jokubaitis V, Izquierdo G, Duquette P, Girard M, Lugaresi A et al. Comparative effectiveness of glatiramer acetate and interferon beta formulations in relapsing - remitting multiple sclerosis. Mult Scler. 2015 Aug;21(9):1159-71.

19. Teter B, Agashivala N, Kavak K, Chouhfeh L, Hashmonay R, Weinstock-Guttman B. Characteristics influencing therapy switch behavior after suboptimal response to first-line treatment in patients with multiple sclerosis. Mult Scler 2013. sclerosis. Mult Scler 2013. Nov 25. [Epub ahead of print].

20. Hillert J. In the coming year we should abandon interferons and glatiramer acetate as first line therapy for MS: no. Mult Scler. 2013;19:26-8.

21. Marriott JJ, O'Connor PW. Lessons learned from long-term multiple sclerosis treatment trials. Mult Scler. 2010;16:1028-30.

22. Jokubaitis VG, Spelman T, Lechner-Scott J, Barnett M, Shaw C, Vucic S, et al. The Australian Multiple Sclerosis (MS) immunotherapy study: a prospective, multicentre study of drug utilisation using the MSBase platform. PLoS One. 2013;8:e59694.

23. Kleinman NL, Beren IA, Rajagopalan K, Brook RA. Medication adherence with disease modifying treatments for multiple sclerosis among US employees. J Med Econ. 2010;13:633-40.

24. Mikol DD, Barkhof F, Chang P, Coyle PK, Jeffery DR, Schwid SR, et al. Comparison of subcutaneous interferon beta-1a with glatiramer acetate in patients with relapsing multiple sclerosis (the REbif vs Glatiramer Acetate in Relapsing MS Disease [REGARD] study): a multicentre, randomised, parallel, open-label trial. Lancet Neurol. 2008;7:903-14.

25. Meyniel C, Spelman T, Jokubaitis VG, Trojano M, Izquierdo G, Grand'Maison F, et al. Country, sex, EDSS change and therapy choice independently predict treatment discontinuation in multiple sclerosis and clinically isolated syndrome. PLoS One. 2012;7:e38661.

26. Portaccio E, Zipoli V, Siracusa G, Sorbi S, Amato MP. Long-term adherence to interferon beta therapy in relapsing-remitting multiple sclerosis. Eur Neurol. 2008;59:131-5.

27. Río J, Porcel J, Téllez N, Sánchez-Betancourt A, Tintoré M, Arévalo MJ, et al. Factors related with treatment adherence to interferon $\beta$ and glatiramer acetate therapy in multiple sclerosis. Mult Scler. 2005;11:306-9.

28. Fox RJ. In the coming year we should abandon interferons and glatiramer acetate as first-line therapy for MS: yes. Mult Scler. 2013;19:24-5.

29. La Mantia L, Di Pietrantonj C, Rovaris M, Rigon G, Frau S, Berardo F, et al. Comparative efficacy of interferon $\beta$ versus glatiramer acetate for relapsing-remitting multiple sclerosis. J Neurol Neurosurg Psychiatry 2014. doi:10.1136/jnnp-2014-309243. [Epub ahead of print]

\section{Submit your next manuscript to BioMed Central and take full advantage of:}

- Convenient online submission

- Thorough peer review

- No space constraints or color figure charges

- Immediate publication on acceptance

- Inclusion in PubMed, CAS, Scopus and Google Scholar

- Research which is freely available for redistribution

Submit your manuscript at www.biomedcentral.com/submit 